\title{
Cepheid Distances to Nearby Galaxies
}

\author{
Wolfgang P. Gieren, Pablo Candia \\ Pontifica Universidad Católica de Chile, Casilla 104, Santiago 22, Chile \\ Pascal Fouqué
}

DESPA Observatoire de Paris-Meudon, F-92195 Meudon Cedex France and European Southern Observatory, Casilla 19001, Santiago 19, Chile

Gisela Hertling

Pontifica Universidad Católica de Chile, Casilla 104, Santiago 22, Chile

\author{
Eduardo Brieva \\ Observatorio Astronómico Nacional, Apartado Aéreo 2584, Bogotá, \\ Colombia
}

\begin{abstract}
We have determined new Cepheid distances on a homogeneous system to nine nearby galaxies with Cepheid observations in the $\mathrm{V}$ bandpass, using the extensive calibration of the galactic Cepheid PL(V) relation of Gieren, Barnes \& Moffett (1993). Absorption corrections to the observed distance moduli were taken from the RC3 catalog (de Vaucouleurs et al. 1991). For most of the galaxies, the galactic PL(V) relation of GBM gives a very good fit to the Cepheid data.
\end{abstract}

\section{Introduction}

Recently Gieren, Barnes \& Moffett (1993; hereafter GBM) have published a new galactic calibration of the Cepheid period-luminosity (PL) relation in the $\mathrm{V}$ bandpass. This relation, based upon the distances of 100 Cepheids derived via the surface brightness method (Barnes \& Evans 1976), is the most extensive calibration of the PL relation currently available. Using this calibration which is assumed to be universal, and applying it to Cepheids in other galaxies for which $\mathrm{V}$ light curves have been observed, a homogeneous set of internally consistent distances for these galaxies can be derived.

\section{Data and distance determination}

We surveyed the literature for galaxies for which $V$ light curves for a substantial number of Cepheid variables are available. This defined a sample of nine galaxies including M81, IC 4182 and NGC 5253 for which Hubble Space Telescope data have recently become available. 
For each of these galaxies we plotted the mean V magnitudes of the Cepheids, as given in the original references, versus the logarithm of the pulsation period. We then fitted the GBM PL relation

$$
\begin{aligned}
M_{\langle V\rangle}= & -1.371-2.986 \log P \\
& \pm 0.095 \pm 0.094
\end{aligned}
$$

to the data. The GBM calibration gives a very good representation of the $\langle V\rangle$ versus $\log P$ diagrams for most, albeit not all, of the studied galaxies. On the basis of these plots, we decided the location of the detection limits for each galaxy and omitted those short-period (faint) Cepheids from the samples which appeared to be affected by the Malmquist bias. For each of the remaining Cepheids in the different galaxy samples, a distance modulus $\left(V-M_{V}\right)$ was calculated where $M_{V}$ came from equation (1). Finally, a mean distance modulus was calculated for each galaxy averaging the individual Cepheid $\mu$ values. In this process, weights of $0,0.5$, and 1 were assigned to the individual Cepheids according to the quality and amplitudes of their light curves. The apparent distance moduli were corrected for interstellar absorption using the $A_{B}$ values given in the RC3 catalog (de Vaucouleurs et al. 1991) which were transformed to absorption in the $\mathrm{V}$ band using $A_{V} / A_{B}=0.78$.

On average the distance moduli we obtain are about $0.3 \mathrm{mag}$ larger than the mean of previous determinations (see de Vaucouleurs 1993). This is partly due to the adopted galactic calibration of the PL(V) relation of GBM, which makes Cepheids 0.2 mag brighter than the Feast \& Walker (1987) calibration from ZAMS-fitting of open clusters, as well as to our adopted absorption corrections. Numerical values will be published elsewhere.

Acknowledgments. This work was supported by FONDECYT grant No. 1940710 given to W. Gieren, and by a Colciencias grant given to the Universidad Nacional de Columbia. Both grants are gratefully acknowledged.

\section{References}

Barnes, T. G. \& Evans, D. S. 1976, MNRAS, 174, 498

De Vaucouleurs, G. 1993, ApJ, 41510

De Vaucouleurs, G., de Vaucouleurs, A., Corwin, H. G., Buta, R. J., Paturel, G., \& Fouqué, P. 1991, Third Reference Catalogue of Bright Galaxies, Vol. II, Springer-Verlag

Feast, M. W. \& Walker, A. R. 1987, ARA\&A, 25, 345

Gieren, W. P., Barnes, T. G. \& Moffett, T. J. 1993, ApJ, 418, 135 